TECHNICAL NOTE

\author{
D.M. Sciubba \\ J.-P. Wolinsky \\ K.D. Than \\ Z.L. Gokaslan \\ T.F. Witham \\ K.P. Murphy
}

\section{CT Fluoroscopically Guided Percutaneous Placement of Transiliosacral Rod for Sacral Insufficiency Fracture: Case Report and Technique}

SUMMARY: Treatment of sacral insufficiency fractures (SIFs) has traditionally been conservative, but several patients have been treated with percutaneous sacroplasty. Unfortunately, in the setting of severe, bilateral SIFs, cement may not withstand shear forces present at the lumbosacral junction, and surgical hardware may not provide adequate fixation in osteoporotic, cancellous bone of the sacrum, leading to eventual pseudarthrosis. Thus, we propose a novel technique in which guidance with CT fluoroscopy allows placement of a transiliosacral bar in conjunction with sacroplasty.

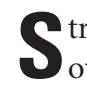
tress fractures of the sacrum may represent commonly overlooked causes of low back pain. Such injuries primarily occur in elderly women with osteoporosis as a result of normal stress on abnormally weak bone. First described by Lourie in 1982 as a "spontaneous fracture of the sacrum," ${ }^{1}$ this injury is known as a sacral insufficiency fracture (SIF). Although most occur in zone 1 of the sacrum, ${ }^{2}$ thus involving the sacral alae without damaging the neural elements in the central canal or foramina, pain can be severe and may significantly limit the patient's mobilization. ${ }^{3}$ Given that the prevalence in female patients older than 55 years in the United States is estimated to be approximately $2 \%,{ }^{4}$ treatment options for such patients must be explored.

Traditional therapy for a SIF has included prolonged bed rest with pain medication followed by physical therapy and medical treatment of osteoporosis. Despite that patients generally note improvement of pain within weeks to months with such treatment, ${ }^{5}$ prolonged bed rest in elderly patients can be associated with significant morbidity. ${ }^{3}$ Moreover, the medical costs imparted by acute inpatient hospitalizations have been estimated to be comparable with those imparted by femoral neck fractures. ${ }^{3}$ Unfortunately, surgical fixation is often technically infeasible because of pullout or fracture of hardware within the weakened bone. As a result, placement of polymethylmethacrylate (PMMA) cement along the sacral fracture planes with the use of image guidance, a technique known as percutaneous sacroplasty, has recently been shown to effectively reduce acute pain in such patients, which allows early mobilization. ${ }^{6-8}$

However, there is concern about the durability of such treatment, given that the stress forces at the lumbosacral junction are more complex than those of the mobile spine. ${ }^{9}$ Specifically, the physiologic transmission of downward forces of the spine on the laterally located ilia creates significant shear

Received February 28, 2007; accepted after revision April 4

From the Departments of Neurosurgery (D.M.S., J.-P.W., K.D.T., Z.L.G., T.F.W.) and Radiology (K.P.M.), Johns Hopkins University School of Medicine, Baltimore, Md.

The senior author (K.P.M.) receives royalties from Cook involving the sale of the Cook Murphy bone biopsy needle.

Please address correspondence to Daniel M. Sciubba, MD, 600 North Wolfe St, Meyer Building 8-161, Baltimore, MD 21237; e-mail: dsciubb1@jhmi.edu

DOI 10.3174/ajnr.A0665 forces at the sacrum, compared with primary compressive forces at the lumbar and thoracic vertebral bodies. Although there are no current reports of failed sacroplasty, PMMA may be less than ideal for such shear forces, which raises concern for eventual pseudarthrosis in patients with severe bilateral SIFs treated with sacroplasty alone. Thus, we present the case of an elderly woman with osteoporosis and SIFs who underwent placement of a transiliosacral rod in conjunction with sacroplasty via guidance with CT fluoroscopy. By combining the benefits of sacroplasty and internal fixation, we hypothesize that a sounder biomechanical construct can be created and still remain minimally invasive.

\section{Case Illustration}

A 76-year-old woman with osteoporosis presented with 8 months of severe low back, pelvic, and buttock pain. The pain was exacerbated by sitting or standing and relieved by assuming a supine position. On a visual analog scale (VAS), the pain was rated as $5 / 10$ with rest and $6-8 / 10$ with walking. The patient's past medical history was notable for anorexia nervosa, hysterectomy, 2 previous lumbar diskectomies, polymyalgia rheumatica, and a long history of smoking. Medications included prednisone, conjugated estrogen supplements, hydroxychloroquine, calcium and vitamin D supplements, and hydrocodone and cyclobenzaprine for chronic pain.

On neurologic examination, the patient was $157.5 \mathrm{~cm}(62$ inches) and weighed $59 \mathrm{~kg}(131 \mathrm{lb})$. Neurologic examination was within normal limits. CT images of the lumbosacral spine demonstrated severe osteoporosis, sacroiliac joint incompetence, and bilateral comminuted fractures of the sacrum and pelvis (Fig 1). There was concern that lone sacroplasty would not provide adequate stabilization of her SIFs, and thus CT fluoroscopically guided percutaneous placement of a transiliosacral rod combined with sacroplasty was recommended. We discussed the potential risks and benefits of the procedure with the patient, as well as the options of lone sacroplasty and conservative medical management. After lengthy discussion, the patient agreed to undergo the proposed procedure.

\section{Technique}

In the operating room, the patient underwent general endotracheal anesthesia and was positioned prone on a Jackson table. She was then transferred to the CT scanner suite (Fig 2). With CT fluoroscopic 


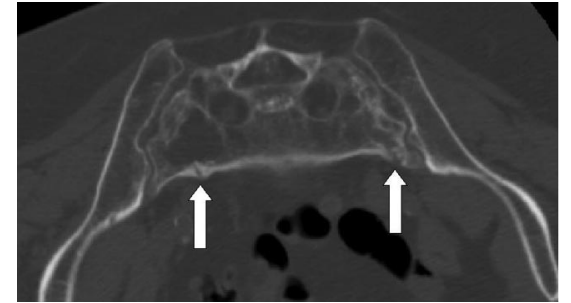

Fig 1. Axial CT image of the sacrum showing severe osteoporosis and bilateral comminuted fractures of the sacral alae.

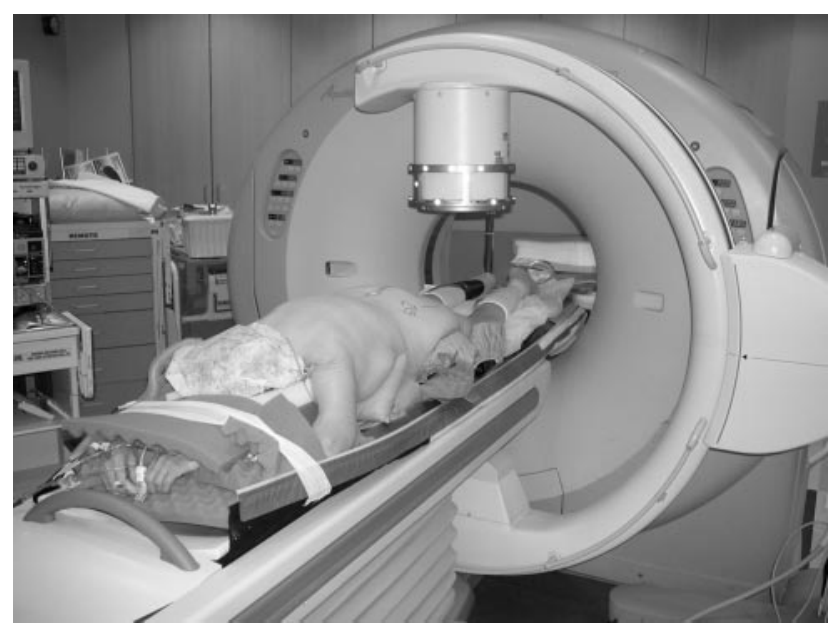

Fig 2. Photograph of the patient in the CT fluoroscopy suite after intubation and positioning on a Jackson table in the operating room.

guidance, a 15-cm-long, 11-gauge Osteo-Site Murphy Quick Set bone biopsy needle (Cook, Bloomington, Ind) was passed from the left ilium at the level of S1, through the left sacroiliac joint, across the anterior body of the sacrum into the right sacral ala and across the right sacroiliac joint (Fig 3). A Kirschner wire (K-wire) was then introduced down each vertebroplasty needle through the inner table of the contralateral bone and across the sacroiliac joint on the other side. The vertebroplasty needle was then removed.

The patient was then transferred back to the operating room. A 4-cm incision was made over each iliac crest. A transsacral cannulated 8-mm rod (Synthes, West Chester, $\mathrm{Pa}$ ) was passed over the $\mathrm{K}$-wire and through the left ilium, left ala, S1 vertebral body, right ala, and right ilium via the trajectory previously determined on the CT scanner. A washer and hexagon nut were secured on both ends of the rod, and the K-wire was removed. The incisions were irrigated and closed. The patient was then transferred to the CT suite, where she received bilateral sacral cement injections with the use of CT-guided fluoroscopy to solidify hardware and index the cement to the fracture planes of the sacral alae (Fig 3). There were no complications, and blood loss was less than $50 \mathrm{~mL}$. The patient awoke without any neurologic deficits, and she rated her pain as 6/10 on the VAS. Immediately postoperatively, the patient used a patient-controlled narcotic pump, which she continued until postoperative day 2 .

Although the patient was allowed to walk on the night of surgery, she was formally assisted for her first ambulation with physical therapy on postoperative day 1 because of concern for falls in an older patient who was ambulating poorly for an extended period of time before the procedure. On discharge, her pain was managed with oral narcotics (14-hydroxydihydrocodeinone). At 2-week and 1-month follow-ups, she was ambulating independently, rating her pain as a
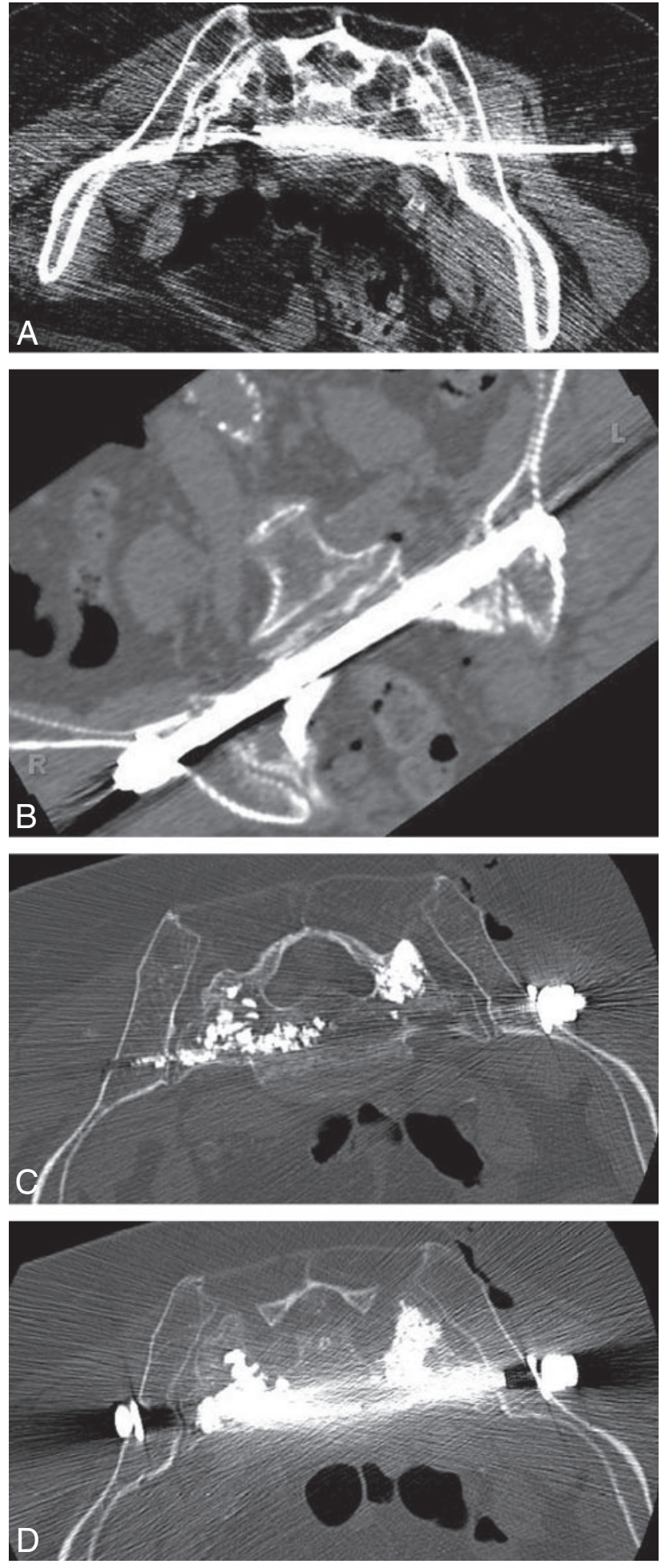

Fig 3. Axial CT images of the sacrum during placement of vertebroplasty needle $(A)$, after placement of transiliosacral rod $(B)$, and after sacroplasty $(C, D)$.

$2-4 / 10$, and progressing well in her rehabilitation program. At 6 months, she complained only of minimal, intermittent pain (1-2/10) after periods of prolonged exercise.

\section{Discussion}

Sacral insufficiency fractures are estimated to occur in nearly $2 \%$ of women who are older than 55 years. ${ }^{4}$ The primary risk factor is postmenopausal osteoporosis followed most com- 
monly by pelvic irradiation, corticosteroid therapy, and rheumatoid arthritis. ${ }^{10}$ Although often caused by relatively minor trauma, ${ }^{5}$ resulting pain can be severe, leading to a bedridden state. Unfortunately, the management of such fractures can be difficult. Patients classically complain of low back, pelvic, and buttock pain, but in elderly patients these complaints can be nonspecific to the sacrum, and thus insufficiency fractures are likely misdiagnosed. ${ }^{10}$ Initial conventional radiologic techniques may not detect such fractures because of limited sensitivity. ${ }^{1}$ As a result, CT, bone scintigraphy, and MR imaging may be required for accurate assessments of anatomy and acuity of fractures. ${ }^{11,12}$ From a traditional standpoint, treatment of sacral insufficiency fractures has included bed rest, pain medication, and treatment with calcium and vitamin $\mathrm{D}$, bisphosphonates, and calcitonin for underlying osteoporosis, ${ }^{5}$ followed by a return to normal activities over a period of months. ${ }^{10,13}$

Patients with conservative management generally experience improvement of symptoms after 1 to 2 weeks of treatment, with most patients pain free 6 to 12 months with complete healing after 9 months. ${ }^{5}$ Unfortunately, such immobility may lead to deep vein thrombosis, pulmonary embolism, pneumonia, impaired cardiac function, and further progression of osteoporosis. ${ }^{3}$ Currently, no effective surgical techniques are available to instrument sacral insufficiency fractures because of technical difficulties in the fixation of osteoporotic, cancellous bone in this region. However, minimally invasive percutaneous techniques such as vertebroplasty, kyphoplasty, and sacroplasty continue to show efficacy in the treatment of painful fractures of the spine with limited morbidity and early mobilization, ${ }^{14,15}$ and thus such options have become more popular.

Originally pioneered by Galibert et $\mathrm{al}^{16}$ in 1987 for treatment of a hemangioma at $\mathrm{C} 2$, percutaneous vertebroplasty has become a commonly used technique for the treatment of osteoporotic fractures, painful metastases to the vertebral bodies, and aggressive hemangiomas of the vertebral bodies. In the pelvis, injection of PMMA cement was initially described for the treatment of painful metastases and fractures that occur in the setting of malignant tumors, but percutaneous sacroplasty for osteoporotic fractures has become a common intervention. ${ }^{17,18}$ The mechanism by which injected cement provides pain relief has mainly been attributed to structural stabilization. ${ }^{19}$ In cases of metastatic fractures, PMMA may also be directly toxic to tumor cells. ${ }^{19,20}$

In relationship to SIF, numerous authors ${ }^{6-8}$ have documented pain relief, improved mobility, and decreased analgesic dependence after biplanar fluoroscopic-guided or CTguided percutaneous injection of PMMA. Brook et $\mathrm{al}^{6}$ has hypothesized that the cement provides fracture stabilization, thus decreasing painful micromotion. Unlike standard vertebroplasty of the mobile spine, the relationship between the cement and sacral foramina can be difficult to ascertain on standard fluoroscopy because of the oblique configuration of the sacrum and its foramina. For this reason, CT guidance allows for improved resolution, which potentially promotes safer placement of cement or hardware near adjacent vascular, neurologic, and visceral structures. ${ }^{6,21}$ In addition, as suggested by Pommersheim et al, ${ }^{8} \mathrm{CT}$ fluoroscopy provides both the precision of CT and the direct visualization of cement de- livery through real-time fluoroscopy. For this reason, we chose to treat our patient via this imaging technique.

Although sacroplasty seems like a reasonable option for such patients, the loading forces at the sacrum are not directly comparable with those placed on the vertebral bodies of the mobile spine. In the lumbosacral region, where the torso transmits a downward force vector that must be distributed laterally to the pelvic ring to maintain painless weightbearing posture, sacral fracture planes are likely to be stressed by significant shear force vectors. Using a finite element model of sacroplasty, Anderson et $\mathrm{al}^{9}$ showed that the sacrum has a $3 \mathrm{D}$ multiaxial state of strain with significant tensile and shear vectors. Unfortunately, although PMMA cement is strong in compression, its long-term strength may be limited under shear force vectors, ${ }^{9}$ because there is histologic evidence that PMMA does adhere to bone. ${ }^{22}$

At present, there are no current reports of failed sacroplasty for the treatment of SIFs, and sacroplasty has been shown to provide long-term improvements in pain and functional recovery. However, in the management of severe bilateral fractures of the sacral alae, as were evident in this patient, it is possible that PMMA may fail to sufficiently stabilize micromotion of the fracture plane, preventing complete bone healing. Likewise, because the modulus of the elasticity of surgical hardware can be 6 to 10 times that of normal bone, ${ }^{23}$ lone surgical fixation would likely be complicated by failure at the hardware-bone interfaces. However, by combining these 2 techniques, the hardware may provide greater stabilization of shear forces, and the cement may provide stabilization between the hardware and the weakened bone.

The ability of PMMA to improve fixation of hardware in osteoporotic bone has been supported by improved pullout strength of pedicle screws in osteoporotic cadaveric bone during biomechanical testing, ${ }^{24,25}$ and, thus, during spinal fixation procedures on such patients, PMMA is often injected immediately before placement of screws. Although not done in this case, such procedures can theoretically be done entirely within the CT suite with techniques of percutaneous CT fluoroscopy, therefore minimizing any increased risk for infection during transport to the operating room. In doing so, the patient may be treated as an outpatient with immediate return to weightbearing activity, which leads to less bed rest-associated morbidity and more cost-effective health care for a rapidly growing elderly population.

In our experience, we recommend an algorithmic approach to the treatment of osteoporotic fractures, including 4 to 6 weeks of conservative management (rest, pain management) for those patients with minimal to moderate pain and minimal functional impairment. For more severely afflicted patients, or for those who fail conservative management, we recommend lone sacroplasty for unilateral fractures that involve either the anterior or posterior cortex. If both cortices are involved, or if the fractures are bilateral, or both, and they lead to a severely destabilized sacrum, augmentation with instrumentation may, in theory, decrease the likelihood of cement failure.

\section{References}

1. Lourie H. Spontaneous osteoporotic fracture of the sacrum. An unrecognized syndrome of the elderly. JAMA 1982;248:715-17

2. Denis F, Davis S, Comfort T. Sacral fractures: an important problem. Retrospective analysis of 236 cases. Clin Orthop Relat Res 1988;227:67-81 
3. Babayev M, Lachmann E, Nagler W. The controversy surrounding sacral insufficiency fractures: to ambulate or not to ambulate? Am J Phys Med Rehabil 2000;79:404-09

4. Weber M, Hasler P, Gerber H. Insufficiency fractures of the sacrum. Twenty cases and review of the literature. Spine 1993;18:2507-12

5. Lin JT, Lane JM. Sacral stress fractures. J Womens Health (Larchmt) 2003;12:879-88

6. Brook AL, Mirsky DM, Bello JA. Computerized tomography guided sacroplasty: a practical treatment for sacral insufficiency fracture: case report. Spine 2005;30:E450-54

7. Garant M. Sacroplasty: a new treatment for sacral insufficiency fracture. J Vasc Interv Radiol 2002;13:1265-67

8. Pommersheim W, Huang-Hellinger F, Baker M, et al. Sacroplasty: a treatment for sacral insufficiency fractures. AJNR Am I Neuroradiol 2003;24:1003-07

9. Anderson DE, Cotton JR. Mechanical analysis of percutaneous sacroplasty using CT image based finite element models. Med Eng Phys 2007;29:316-25

10. Grasland A, Pouchot J, Mathieu A, et al. Sacral insufficiency fractures: an easily overlooked cause of back pain in elderly women. Arch Intern Med 1996;156:668-74

11. Ries T. Detection of osteoporotic sacral fractures with radionuclides. Radiology 1983;146:783-85

12. Cleary K, Clifford M, Stoianovici D, et al. Technology improvements for image-guided and minimally invasive spine procedures. IEEE Trans Inf Technol Biomed 2002;6:249-61

13. Newhouse KE, el-Khoury GY, Buckwalter JA. Occult sacral fractures in osteopenic patients. J Bone Joint Surg Am 1992;74:1472-77

14. Mathis JM, Barr JD, Belkoff SM, et al. Percutaneous vertebroplasty: a developing standard of care for vertebral compression fractures. AJNR Am J Neuroradiol 2001;22:373-81
15. Watts NB, Harris ST, Genant HK. Treatment of painful osteoporotic vertebral fractures with percutaneous vertebroplasty or kyphoplasty. Osteoporos Int 2001;12:429-37

16. Galibert P, Deramond H, Rosat P, et al. [Preliminary note on the treatment of vertebral angioma by percutaneous acrylic vertebroplasty]. Neurochirurgie 1987;33:166-68

17. Dehdashti AR, Martin JB, Jean B, et al. PMMA cementoplasty in symptomatic metastatic lesions of the $\mathrm{S} 1$ vertebral body. Cardiovasc Intervent Radiol 2000;23:235-37

18. Marcy PY, Palussiere J, Descamps B, et al. Percutaneous cementoplasty for pelvic bone metastasis [published erratum appears in Support Care Cancer 2000;8:510]. Support Care Cancer 2000;8:500-03

19. Dean JR, Ison KT, Gishen P. The strengthening effect of percutaneous vertebroplasty. Clin Radiol 2000;55:471-76

20. Lyles KW. Management of patients with vertebral compression fractures. Pharmacotherapy 1999;19:21S-24S

21. Gangi A, Kastler BA, Dietemann JL. Percutaneous vertebroplasty guided by a combination of CT and fluoroscopy. AJNR Am J Neuroradiol 1994;15:83-86

22. Freeman MA, Bradley GW, Revell PA. Observations upon the interface between bone and polymethylmethacrylate cement. J Bone Joint Surg $\mathrm{Br}$ 1982;64:489-93

23. Williamson MB Jr, Aebi M. Biomechanics of the spine and spinal instrumentation. In: Aebi M, Thalgott JS, Webb JK, eds. AO ASIF Principles in Spine Surgery. Berlin: Springer-Verlag; 1998:3-12

24. Sarzier JS, Evans AJ, Cahill DW. Increased pedicle screw pullout strength with vertebroplasty augmentation in osteoporotic spines. J Neurosurg 2002;96(3 Suppl):309-12

25. Cook SD, Salkeld SL, Stanley T, et al. Biomechanical study of pedicle screw fixation in severely osteoporotic bone. Spine J 2004;4:402-08 